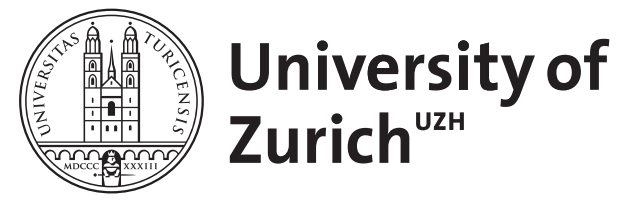

Zurich Open Repository and Archive

University of Zurich

University Library

Strickhofstrasse 39

CH-8057 Zurich

www.zora.uzh.ch

Year: 2013

\title{
Strategien gegen Polypharmazie
}

Neuner-Jehle, S

Posted at the Zurich Open Repository and Archive, University of Zurich ZORA URL: https://doi.org/10.5167/uzh-93676

Journal Article

Published Version

Originally published at:

Neuner-Jehle, S (2013). Strategien gegen Polypharmazie. PrimaryCare, 13(21):377. 


\section{Strategien gegen Polypharmazie}

Ein Beitrag aus der Reihe «Die Werkzeugkiste des Familienarztes»

Polypharmazie - definiert als fünf oder mehr gleichzeitig eingesetzte Medikamente beim gleichen Patienten - ist verbunden mit einem erhöhten Risiko für unerwünschte Ereignisse, Hospitalisation und Tod [1]. In unserer Ära der zunehmenden Anzahl polymorbider Patienten wird sie zum Problem, besonders bei älteren Menschen. Strategien dagegen sind dringend nötig.

Erste Ansätze in Form von Negativlisten (zu vermeidende Medikamente) wurden vor 40 Jahren von Beers und Kollegen entwickelt, seither mehrfach revidiert [2] und später im deutschen Sprachraum für Medikamente weiterentwickelt, die auf unserem Pharma-Markt gebräuchlicher sind (Priscus-Liste) [3]. Ein Blick auf solche Listen ist zwar durchaus lohnenswert - besonders mit dem Fokus auf ältere Patienten -, grosse Verbreitung haben sie aber nicht gefunden. Auch schon länger bekannt ist der medication appropriateness index MAl [4], der für jedes Medikament anhand von zehn Kriterien kritisch hinterfragt, ob der Einsatz in dieser Situation für diesen Patienten adäquat sei. In jüngster Zeit wurde dieser gedankliche Ansatz im geriatrischen Bereich weiterentwickelt und vereinfacht: Der Good Palliative Geriatric Practice (GPGP) - Algorithmus, [5] siehe Kasten. Dieser Algorithmus besteht nur noch aus vier bis fünf Schlüsselfragen und besticht durch seine Einfachheit. Aktuell testen wir am Institut für Hausarztmedizin Zürich, ob er auch in der hausärztlichen Praxis und deren PatientInnen erfolgreich anwendbar sei (Pilottest abgeschlossen, randomisierte Studie in Planung). Ein interessanter, aber wieder etwas komplexerer Ansatz sind die STOPP- und START-Listen aus Irland [6, 7]. Gerade letztere trägt dem Umstand Rechnung, dass viele polypharmazierte Patienten gleichzeitig unter- oder fehlversorgt sind - eine paradoxe Beobachtung, die uns zeigt, dass es mit dem Zusammenstreichen von Medikamentenlisten alleine nicht getan ist.

Schlüsselfragen des GPGP-Algorithmus (vereinfacht):

1 Ist die Indikation korrekt (in Berücksichtigung der Lebenssituation des Patienten)?

2 Bestehen potentielle oder schon vorhandene Nebenwirkungen?

3 Ist die Dosis korrekt für die Situation des Patienten (Nierenfunktion, Alter)?

4 Gibt es eine besser verträgliche oder wirksamere Alternative?

\section{Fazit}

Kurzgefasste Anleitungen zur Reduktion von Polypharmazie, in Anlehnung an den GPGP-

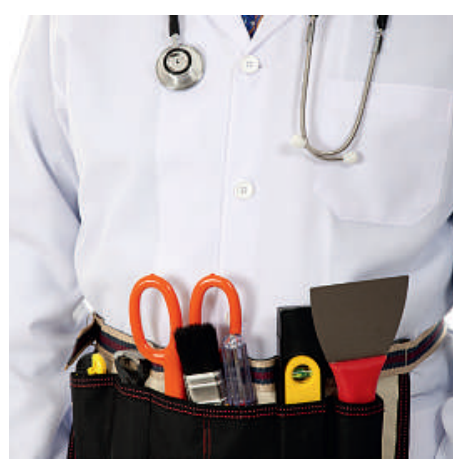
Algorithmus, sind erfolgsversprechend. Das Zauberwort heisst Priorisieren, denn nicht jede Diagnose und jedes Symptom muss beim polymorbiden Patienten behandelt werden. Dieses Priorisieren kann nur zusammen mit dem Patienten erfolgreich umgesetzt werden (shared decision making). Dabei braucht es ein sensibles Vorgehen, damit sich der Patient durch das «Streichkonzert» nicht benachteiligt, entwertet oder gar aufgegeben fühlt, im Sinn von «eine Behandlung lohnt sich nicht mehr für mich» [8].

\section{Literatur}

1 Budnitz DS, Lovegrove MC, Shehab N, Richards CL. Emergency hospitalizations for adverse drug events in older Americans. N Engl J Med. 2011;365:2002-2012.

2 Fick DM, Cooper JW, Wade WE, Waller JL, Maclean JR, Beers MH. Updating the Beers criteria for potentially inappropriate medication use in older adults: results of a US consensus panel of experts. Archives Intern Med. 2003; 163:2716-2724

3 Holt S, Schmiedl S, Thürmann PA. Potentially inappropriate medications in the elderly: the PRISCUS list. Dtsch Ärztebl Int. 2010;107:543-551; www.priscus.net.

4 Hanlon JT, et al. A randomized, controlled trial of a clinical pharmacist intervention to improve inappropriate prescribing in elderly outpatients with polypharmacy. Am J Med. 1996;100:428-437.

5 Garfinkel D, Mangin D. Feasibility study of a systematic approach for discontinuation of multiple medications in older adults: addressing polypharmacy. Arch Intern Med. 2010;170:1648-1654.

6 Gallagher P, Ryan C, Byrne S, Kennedy J, O'Mahony D. STOPP (Screening Tool of Older Person's Prescriptions) and START (Screening Tool to Alert doctors to Right Treatment). Consensus validation. Int J Clin Pharm Therapy. 2008;46:72-83.

7 Barry PJ, Gallagher P, Ryan C, O'mahony D. START (screening tool to alert doctors to the right treatment)--an evidence-based screening tool to detect prescribing omissions in elderly patients. Age Ageing. 2007;36:632-638.

8 Schuling J, et al. Deprescribing medication in very elderly patients with multimorbidity: the view of Dutch GPs. A qualitative study. BMC Fam Pract. 2012;13:56.

\section{Übersichtsarbeiten zum Thema}

- Neuner-Jehle S. Weniger ist mehr - wie Polypharmazie vermeiden? PRAXIS. 2013;02(1):21-27.

- Neuner-Jehle S. Zuviel des Guten - Rezepte gegen Polypharmazie. PrimaryCare. 2011;11(12):212-15.

\section{Korrespondenz:}

Dr. med. Stefan Neuner-Jehle, MPH

Institut für Hausarztmedizin Zürich

Pestalozzistrasse 24

8091 Zürich

sneuner[at]bluewin.ch 